Supplementary Material for

\title{
Direct observation of ring polymer dynamics in the flow-gradient plane of shear flow
}

\author{
Michael Q. Tu ${ }^{1,2}$, Megan Lee ${ }^{3}$, Rae M. Robertson-Anderson ${ }^{3}$, and Charles M. \\ Schroeder ${ }^{1,2,4}$ \\ ${ }^{1}$ Department of Chemical and Biomolecular Engineering, University of Illinois at \\ Urbana-Champaign, Urbana, Illinois 61801, United States \\ ${ }^{2}$ Beckman Institute for Advanced Science and Technology, University of Illinois at \\ Urbana-Champaign, Urbana, Illinois 61801, United States \\ ${ }^{3}$ Department of Physics and Biophysics, University of San Diego, San Diego, California 92110, \\ United States \\ ${ }^{4}$ Department of Materials Science and Engineering, University of Illinois at Urbana-Champaign, \\ Urbana, Illinois 61801, United States
}

\section{Shear rate determination}

The shear rates in the custom-built apparatus are determined via particle tracking velocimetry (PTV) using dilute solutions of tracer particles (Spherotech, $0.84 \mu \mathrm{m}$ diameter). ${ }^{1}$ The imaging setup and imaging parameters are identical to those listed in the main text. Figure 1d in the main text shows a representative set of bead trajectories as viewed in the shear apparatus. Using flow-gradient visualization, the velocity gradient is readily apparent. To determine the shear rate, the fluid velocity in the flow direction (1-direction or $x$-direction) $v_{x}$ is plotted against the gradient coordinate position $y$, thereby enabling determination of shear rate $\dot{\gamma}$, as shown in Figure S1.

$$
\dot{\gamma}=\frac{d v_{x}}{d y}
$$

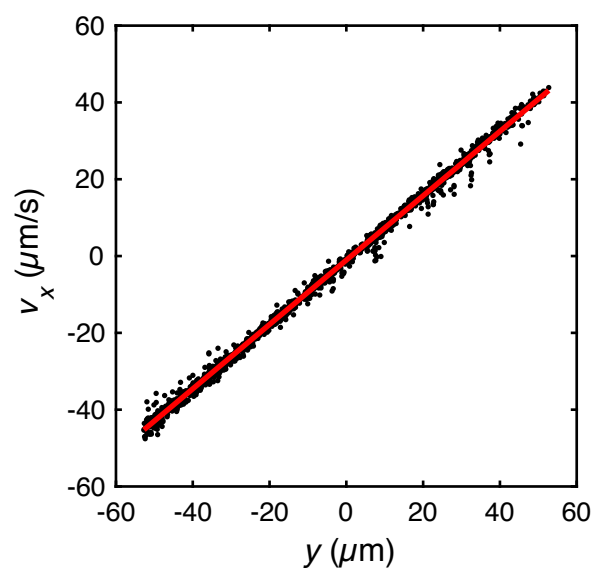

Figure S1: Flow-direction velocity in shear for each trajectory plotted as a function of the gradient position $y$ for the particle tracking velocimetry (PTV) results at $\dot{\gamma}_{\text {applied }}=0.87 \mathrm{~s}^{-1}$. The observed shear rate $\left(0.84 \mathrm{~s}^{-1}\right)$ is determined from the slope of the plot. 


\section{Device characterization}

Due to the no-slip boundary condition at the viewing window coverslip (located at the bottom of the fluid bath in the apparatus), the flow field between the two shearing plates is only fully developed simple shear at a sufficient distance above the bottom surface. Figure $\mathbf{S 2}$ shows the apparent shear rate as a function of the distance $z$ above the coverslip viewing window, determined by manually adjusting the microscope focus. The working distance of the objective used in these experiments is $1.5 \mathrm{~mm}$. All polymer molecules in this work were observed at $z>200 \mu \mathrm{m}$, which is well within the fully developed region of shear flow.

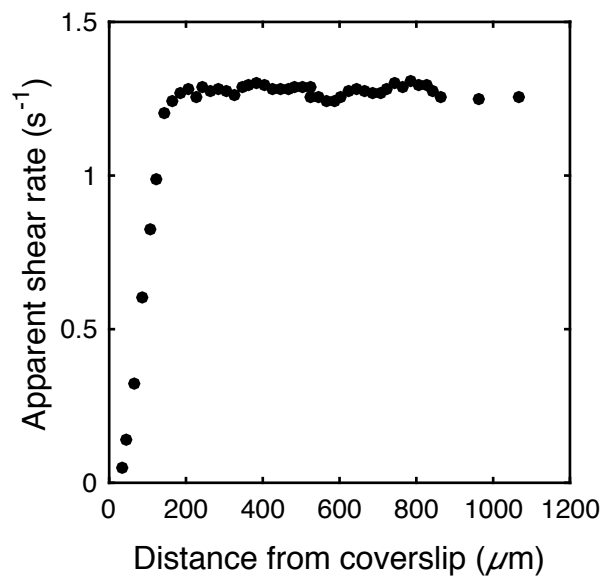

Figure S2: Observed shear rate as a function of the distance $z$ above the coverslip viewing window at $\dot{\gamma}_{\text {applied }}=1.30 \mathrm{~s}^{-1}$. 


\section{Longest relaxation time}

We compare the long relaxation times of the DNA used in this work (48.5 kbp linear and $114.8 \mathrm{kbp}$ ring DNA) to prior work performed on the relaxation of DNA as a function of molecular weight. ${ }^{2}$ In general, the relaxation times obtained in the present work are in excellent agreement with the relaxation times from Li et al. ${ }^{2}$ (Figure S3) when normalized by solution viscosities. As noted in prior work, ring polymers exhibit a smaller exponent power-law relaxation time scaling with molecular weight compared to linear polymers. ${ }^{2,3}$ The relaxation time of ring DNA at a given molecular weight is approximately 2-3 times less than that of the corresponding linear DNA, which deviates from the expected 4-fold difference as predicted by Rouse theory. ${ }^{3,4}$ This difference is not surprising, as Rouse theory does not incorporate hydrodynamic effects that affect the relaxation dynamics of dilute polymers in solution.

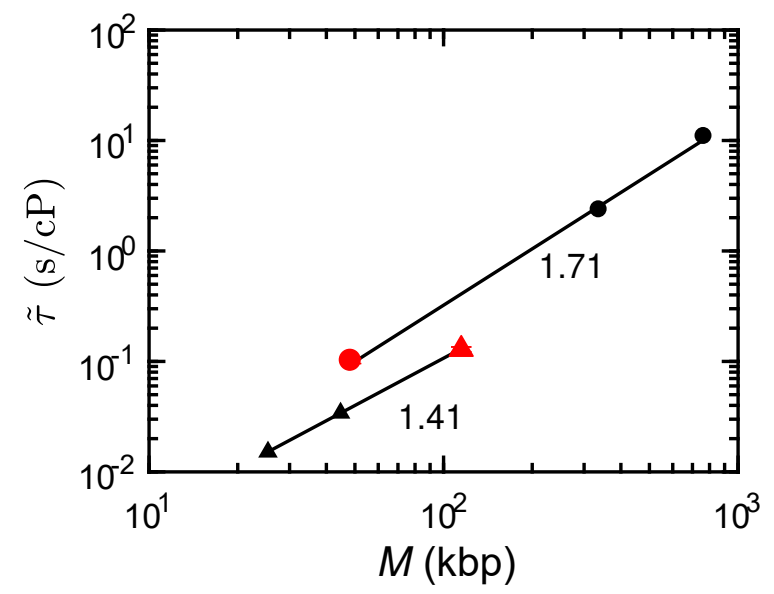

Figure S3: Longest relaxation times scaled by solution viscosity $(\tilde{\tau}=\tau / \eta)$ of linear (circles) and ring DNA (triangles). Data from Li $e$ t al. ${ }^{2}$ are shown in black, and data used in the present work are plotted in red. Error bars are smaller than the size of the symbols. 


\section{Ensemble-averaged fractional extension and orientation angles}

Here we compare the results from single molecule experiments on linear and ring DNA in shear flow with prior experiments of linear DNA in shear flow. ${ }^{5,6}$
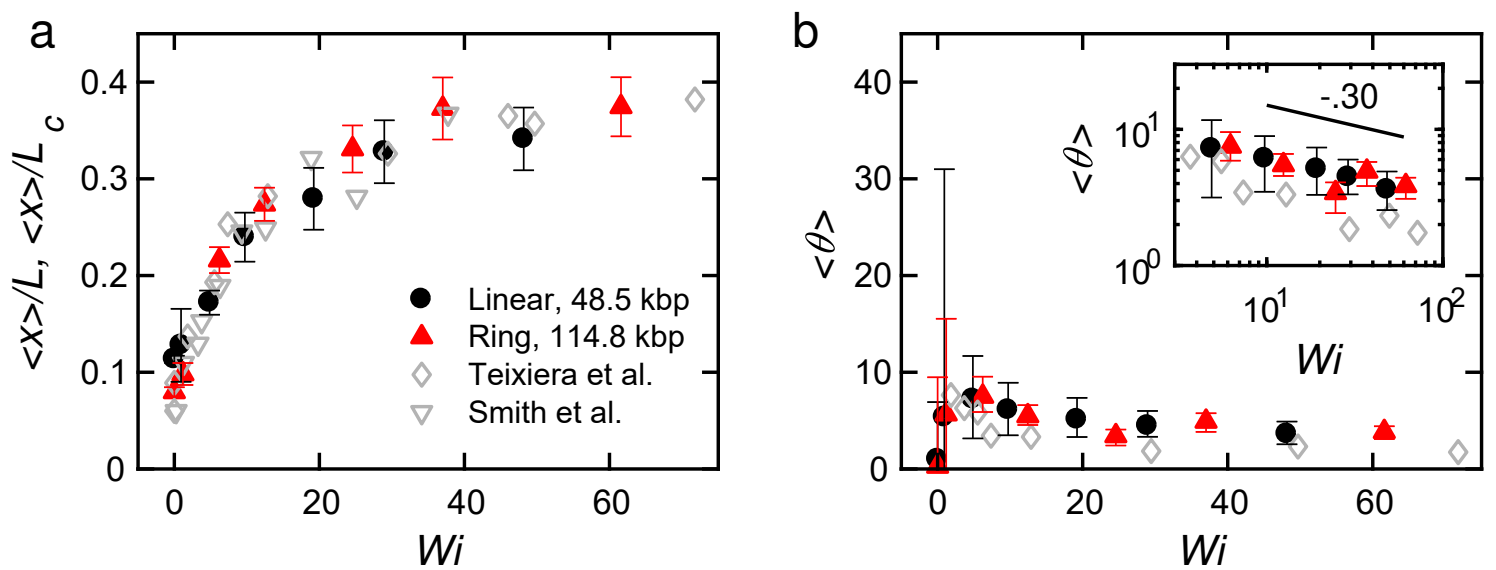

Figure S4: (a) Ensemble-averaged fractional extension as a function of the flow strength in shear flow for rings and linear chains. Prior single molecule data on linear DNA in the flow-gradient (FG) ${ }^{6}$ and flow-vorticity (FV) ${ }^{5}$ are provided for comparison. (b) Ensemble-averaged orientation angle as a function of $W i$. Inset: log-log depiction showing power-law dependence of $<\theta>$ on $W i$. 


\section{Fractional extension distributions at matched Wi}

The probability distributions of fractional extension from BD simulations of linear $\left(N_{k}=160\right)$ and ring polymers $\left(N_{k}=180\right)$ at similar $W i$ are plotted to understand the effect of polymer architecture in Figure S5. Over the range of $W i$ shown, the maxima in the probability distributions of fractional extension for ring polymers occur at larger values than the corresponding linear chains. Moreover, probability distributions for rings are generally more symmetric. This qualitative difference in the shape of the distributions is also observed from BD simulations of rings with $N_{k}=380$, as shown in the main text.

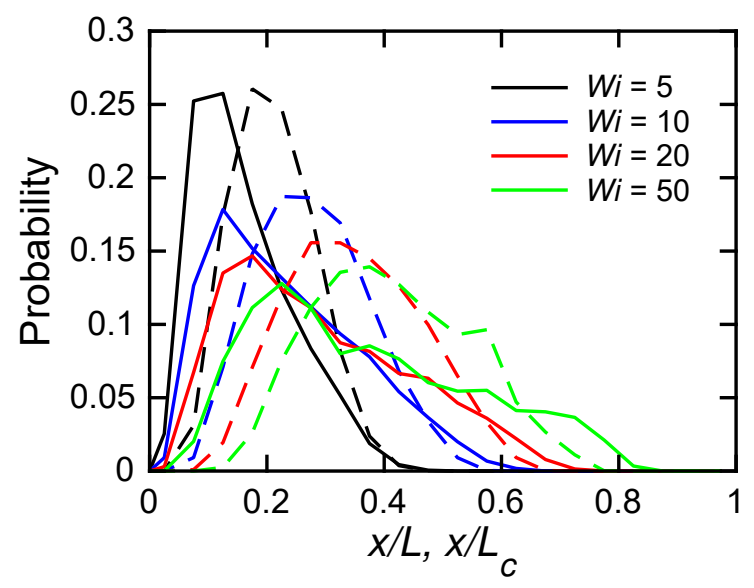

Figure S5: Probability distributions of fractional extension from BD simulations of linear chains (solid lines) and rings (dotted lines) with 160 and 180 Kuhn steps, respectively, as a function of $W i$. 


\section{Supplementary movie}

Movie S1: K16 ring DNA visualized in the flow-gradient plane of shear flow at $W i=6.2$. The movie is played back at $5 x$ speed. Several tumbling events for the single K16 ring polymer are observed. For display purposes, each frame in the movie was filtered to remove background intensity using a Gaussian kernel with a radius of 2 pixels, and false color was applied. 


\section{References}

[1] Anthony, S.; Zhang, L.; Granick, S. Methods to track single-molecule trajectories. Langmuir 2006, 22, 5266-5272.

[2] Li, Y.; Hsiao, K.-W.; Brockman, C. A.; Yates, D. Y.; Robertson-Anderson, R. M.; Kornfield, J. A.; San Francisco, M. J.; Schroeder, C. M.; McKenna, G. B. When Ends Meet: Circular DNA Stretches Differently in Elongational Flows. Macromolecules 2015, 48, 5997-6001.

[3] Hsiao, K.-W.; Schroeder, C. M.; Sing, C. E. Ring Polymer Dynamics Are Governed by a Coupling Between Architecture and Hydrodynamic Interactions. Macromolecules 2016, 49, 1961-1971.

[4] Tsolou, G.; Stratikis, N.; Baig, C.; Stephanou, P. S.; Mavrantzas, V. G. Melt structure and dynamics of unentangled polyethylene rings: Rouse theory, atomistic molecular dynamics simulation, and comparison with the linear analogues. Macromolecules 2010, 43, 10692-10713.

[5] Smith, D. E.; Babcock, H. P.; Chu, S. Single-Polymer Dynamics in Steady Shear Flow. Science 1999, 283, 1724-1727.

[6] Teixeira, R. E.; Babcock, H. P.; Shaqfeh, E. S.; Chu, S. Shear Thinning and Tumbling Dynamics of Single Polymers in the Flow-Gradient Plane. Macromolecules 2005, 38, 581-592. 